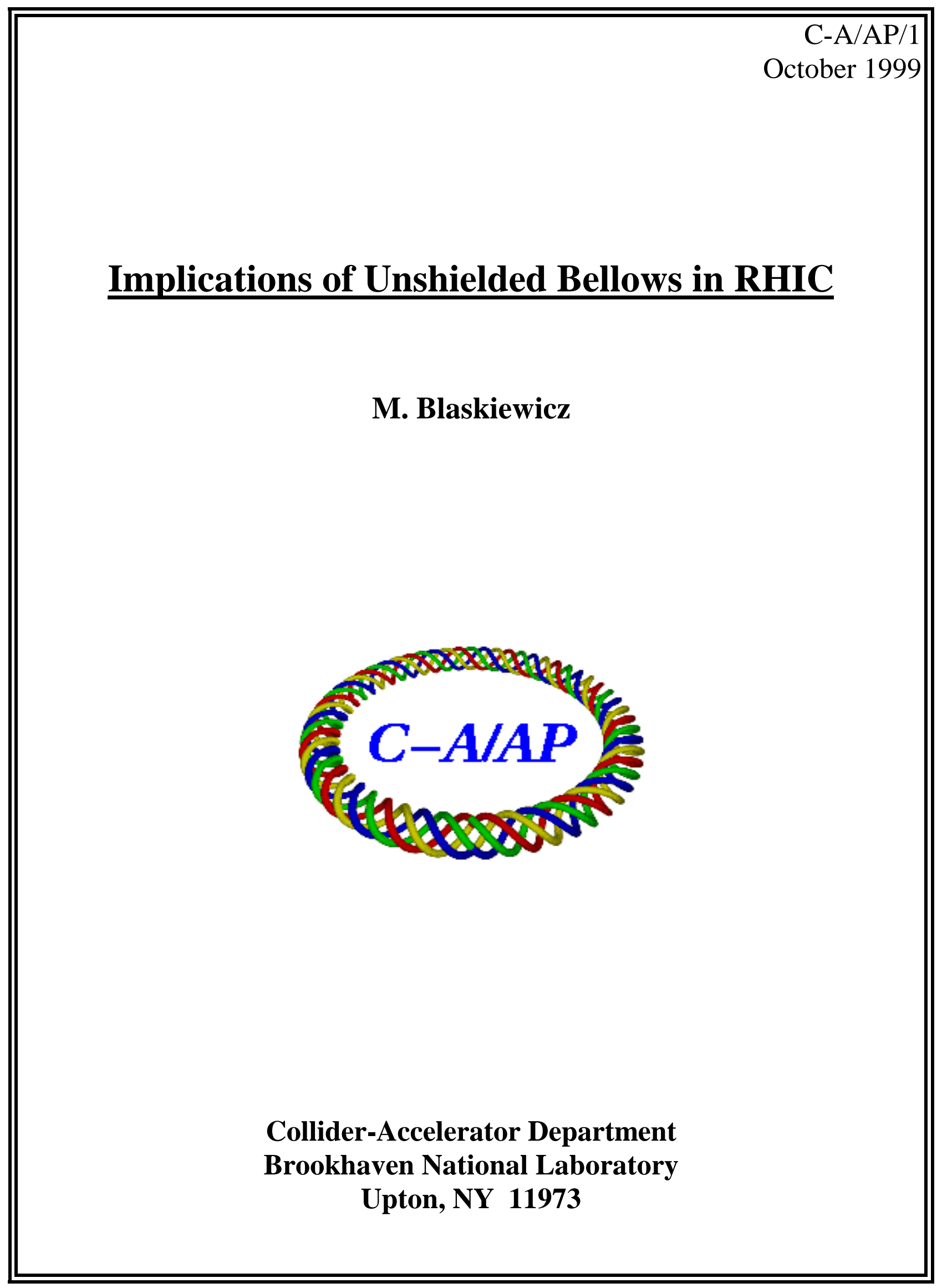




\title{
Implications of unshielded bellows in RHIC
}

\author{
Michael Blaskiewicz \\ CAD Brookhaven National Laboratory
}

\begin{abstract}
The implications of unshielded bellows in RHIC are discussed. It is found that unshielded bellows have a marginal impact on the impedance budget and will increase the heat load on the cryogenic system by about 10 watts.
\end{abstract}

\section{Longitudinal Results}

The model of the bellows used in the estimate is shown in Figure 1. The ABCI[1] simulations used a longitudinal bin size of $\Delta s=0.25 \mathrm{~mm}$ and a radial bin size of $1.0 \mathrm{~mm}$. This corresponds to a $10 \times 10$ grid in each bellows corrugation. The longitudinal and transverse wake potentials were calculated using a Gaussian pulse of rms length $\sigma_{s}=1 \mathrm{~cm}$, which is short compared to the minimum length expected in RHIC; $\sigma_{s} \approx 10 \mathrm{~cm}$. The first meter of the longitudinal wake and the current pulse used are shown in Figure 2.

The Fourier transform of the wake potential is the impedance. The imaginary part of the longitudinal impedance (the longitudinal reactance) for the 24 and 30 corrugation bellows are shown in Figure 3 along with the impedance resulting from an inductance corresponding to $Z_{\|} / n=2 \mathrm{~m} \Omega$. The numerically obtained curves are nearly straight lines below $2 \mathrm{GHz}$, which agrees with measurements [2]. The real part of the longitudinal impedance (the longitudinal resistance) for the 24 and 30 corrugation bellows are shown in Figure 4. Note that the cutoff frequency for microwave propagation in a pipe of radius $3.4 \mathrm{~cm}$ is $3.4 \mathrm{GHz}$.

To estimate heating and dynamical effects two models of the beam will be used; a Gaussian pulse of rms length $10 \mathrm{~cm}$ and a current pulse of the form

$$
I(t)=I_{p}\left[1-t^{2} / \hat{\tau}^{2}\right]^{2}
$$

where $I=0$ for $|t|>\hat{\tau}$ and $\hat{\tau}$ is chosen so that the rms length is $10 \mathrm{~cm}$. The second model is in fair agreement with observations of longitudinal spectra in the AGS. 


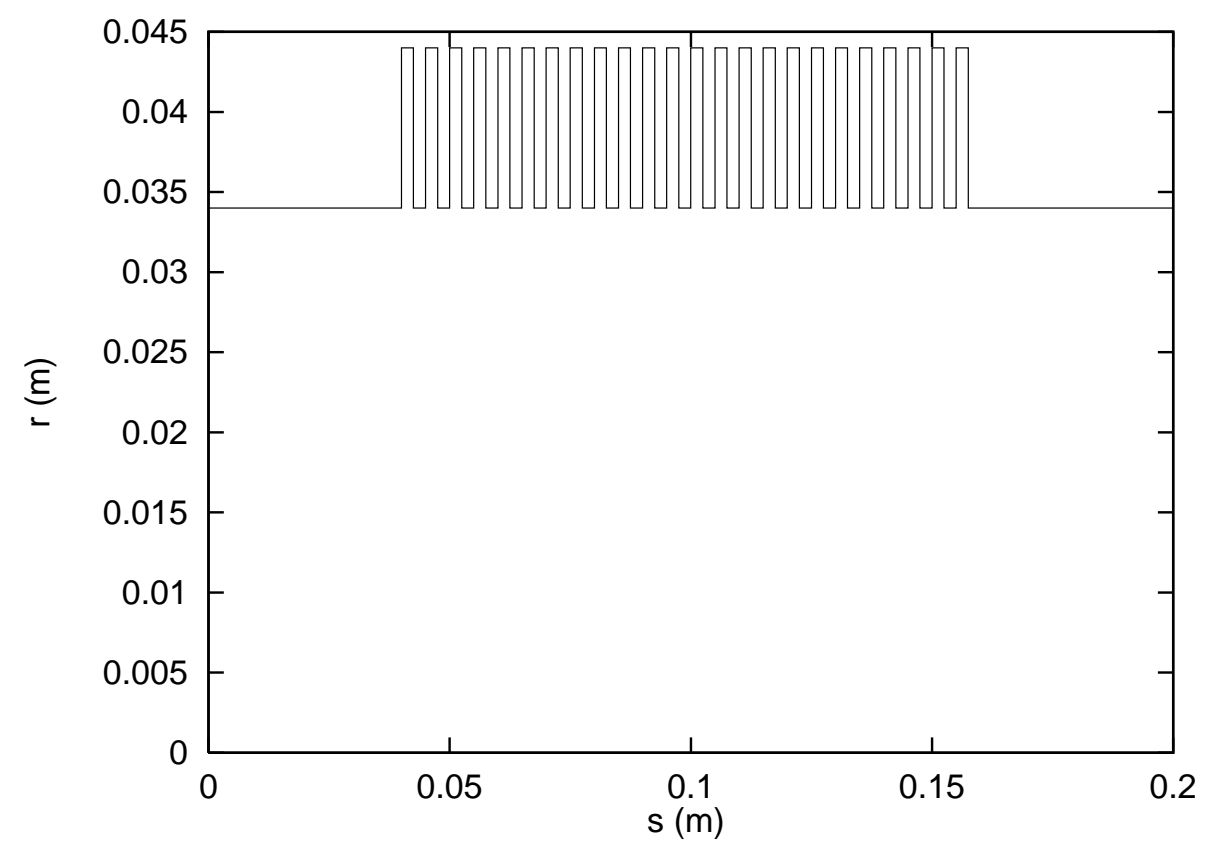

Figure 1: The geometry used in the ABCI calculations for 24 bellows corrugations. Simulations with 30 corrugations where also done.

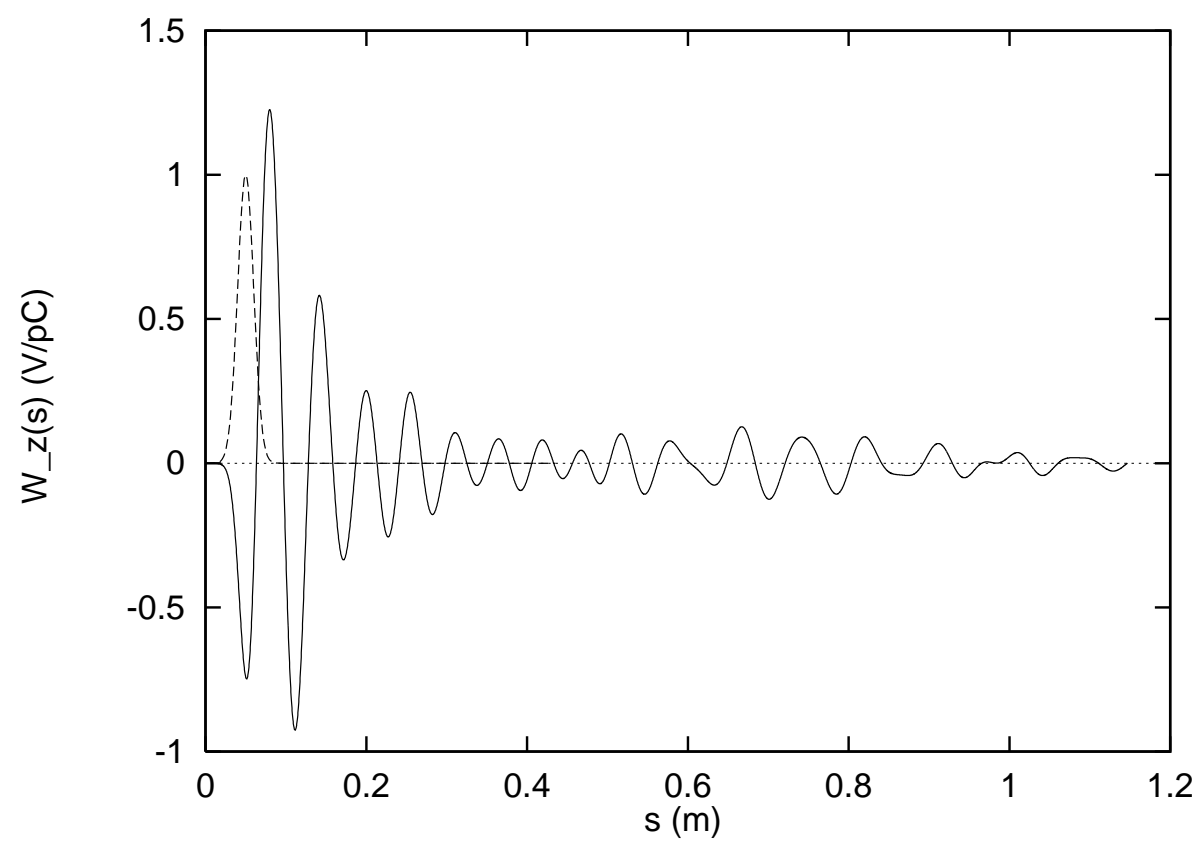

Figure 2: Current pulse and resulting longitudinal wake field for the geometry shown in Figure 1. The actual simulation went out to 10 meters of lag. 


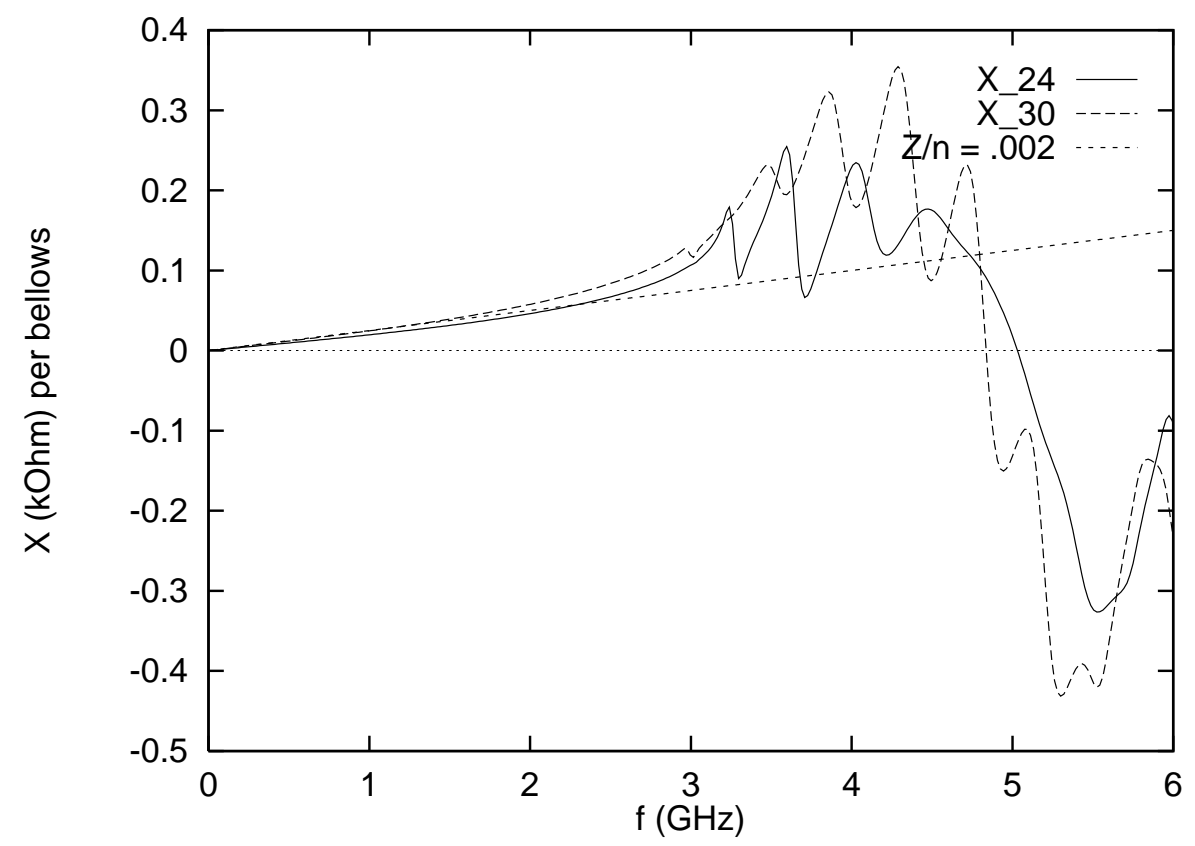

Figure 3: Longitudinal reactance for the 24 and 30 corrugation bellows models and a pure reactance with $Z_{\|} / n=2 \mathrm{~m} \Omega$.

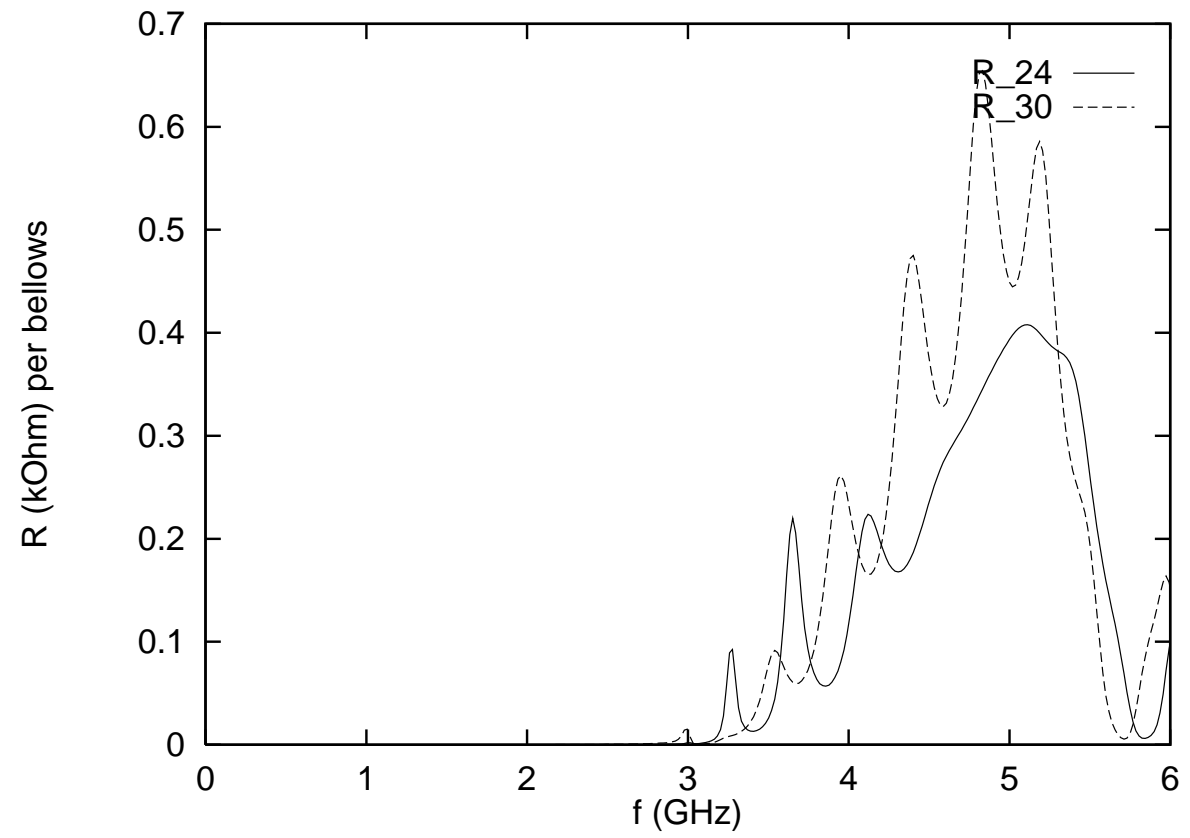

Figure 4: Longitudinal resistance for the 24 and 30 corrugation bellows models. The cutoff frequency for microwave propagation in a pipe of radius $3.4 \mathrm{~cm}$ is $3.4 \mathrm{GHz}$ 


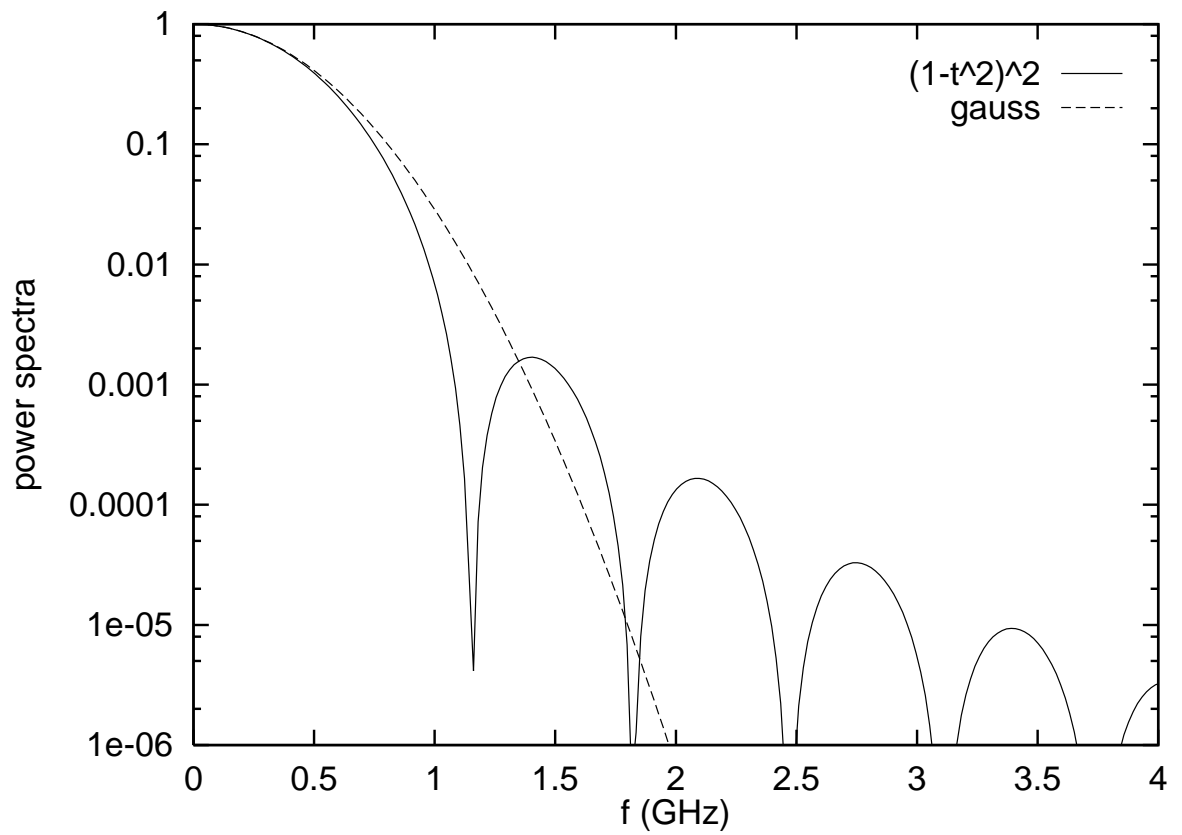

Figure 5: Power spectra for Gaussian current pulse and $I(t)=I_{p}\left[1-t^{2} / \hat{\tau}^{2}\right]^{2}$. Both have a $10 \mathrm{~cm}$ rms bunch length $(1 / 3 \mathrm{~ns}$ rms duration $)$.

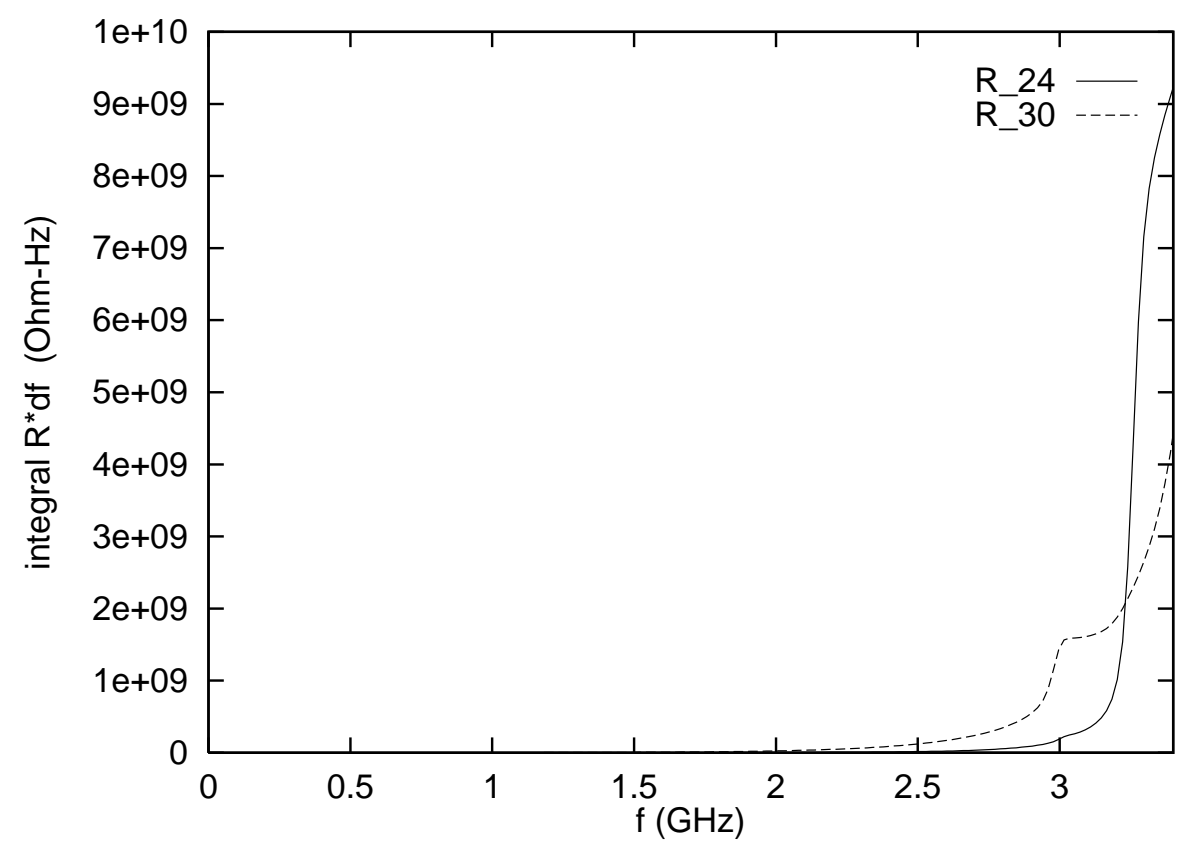

Figure 6: Integrals of resistance with respect to frequency used for estimating the $R / Q$ values of trapped modes. 
The absolute value squared of the Fourier transforms of these current pulses are shown in Fig 5. Note that the decay rate in frequency of the envelope of the solid line is essentially determined by how smoothly the pulse goes to zero in time.

Dynamical effects require that the bunch power spectra significantly overlap the impedance. Since both spectra are down by a factor of at least $10^{4}$ by $2 \mathrm{GHz}$ only frequencies below about $2 \mathrm{GHz}$ are dynamically relevant. For longitudinal dynamics the bellows are pure inductors. The value of $Z_{\|} / n=2 \mathrm{~m} \Omega$ corresponds to $Z_{\|} / n=0.2 \Omega$ for 100 unshielded bellows per ring. Estimates of longitudinal microwave instability set a limit of $Z_{\|} / n=2 \Omega$ and the stripline beam position monitors contribute $Z_{\|} / n=0.58 \Omega[3]$. It should be noted that a pure inductance with a parabolic current pulse is one of the few exactly solvable problems in instabilty theory. Starting with nearly matched initial conditions a pure inductance never causes instability [4]. The main dynamical effect of unshielded bellows will probably be seen around transition. The reactance and the longitudinal space charge impedance will cause a longitudinal mismatch across transition which may require some sort of active damping. This is not unforseen and since the bellows are a small addition to the BPMs it is not an issue.

Heating effects are more subtle. The problem splits into two main parts. The first part involves the trapped modes with resonant frequencies below pipe cutoff. The simulation code reliably predicts the values of $R_{s h} / Q$ for these modes. The values of $R_{s h} / Q$ are found by integrating $R(f)$ with respect to frequency over the numerically induced line width. The values of these integrals for frequencies below pipe cutoff are shown in Figure 6 . The maximum value of these integrals is $\approx$ $10 \Omega \mathrm{GHz}$. If this integral is ascribed to a single resonance then the parameters of the resonance satisfy $R_{s h} \omega_{r} /(4 Q)=$ integral, where $\omega_{r}$ is the angular frequency of the resonance. Setting $\omega_{r}=2 \pi \times 3 \mathrm{GHz}$ yields $R_{s h} / Q=2.1 \Omega$ and $0.32 \Omega$ for the 24 and 30 convolution cases, respectively. The quality factor for the resonance is estimated using the scaling $Q=r_{\text {char }} / \delta_{\text {skin }}\left(\omega_{r}\right)$, where $r_{\text {char }}$ is the characteristic transverse dimension and $\delta_{\text {skin }}\left(\omega_{r}\right)$ is the skin depth evaluated at the resonant frequency [5]. Taking a resistivity of $70 \mu \Omega \mathrm{cm}[6]$ the resulting estimate is $Q \approx 2600$. If anything, this is an overestimate, which will overestimate the heating. Multiplying $R_{s h} / Q$ by $Q$ yields a shunt impedance of $R_{s h}=5.5 \mathrm{k} \Omega$. In the worst case the resonant frequency will be a multiple of the bunching frequency. This is assumed since the code and its input are not reliable at this level. The resulting power dissipation is given by

$$
P_{\text {res }}=2 I_{d c}^{2} R_{s h} F_{b}\left(f_{r}\right),
$$

where $I_{d c}$ is the dc beam current and $F_{b}\left(f_{r}\right)$ is the form factor shown in Figure 5 , evaluated at the resonant frequency. For 120 bunches with $2 \times 10^{11}$ charges each $I_{d c}=0.30 \mathrm{~A}$. Using the envelope of the larger curve in Figure 5 gives $F_{b}\left(f_{r}\right)=$ $2 \times 10^{-5}$. The net power is $P_{\text {res }}=20 \mathrm{~mW}$.

Along with the power dissipated in the narrow band resonance there is the power 


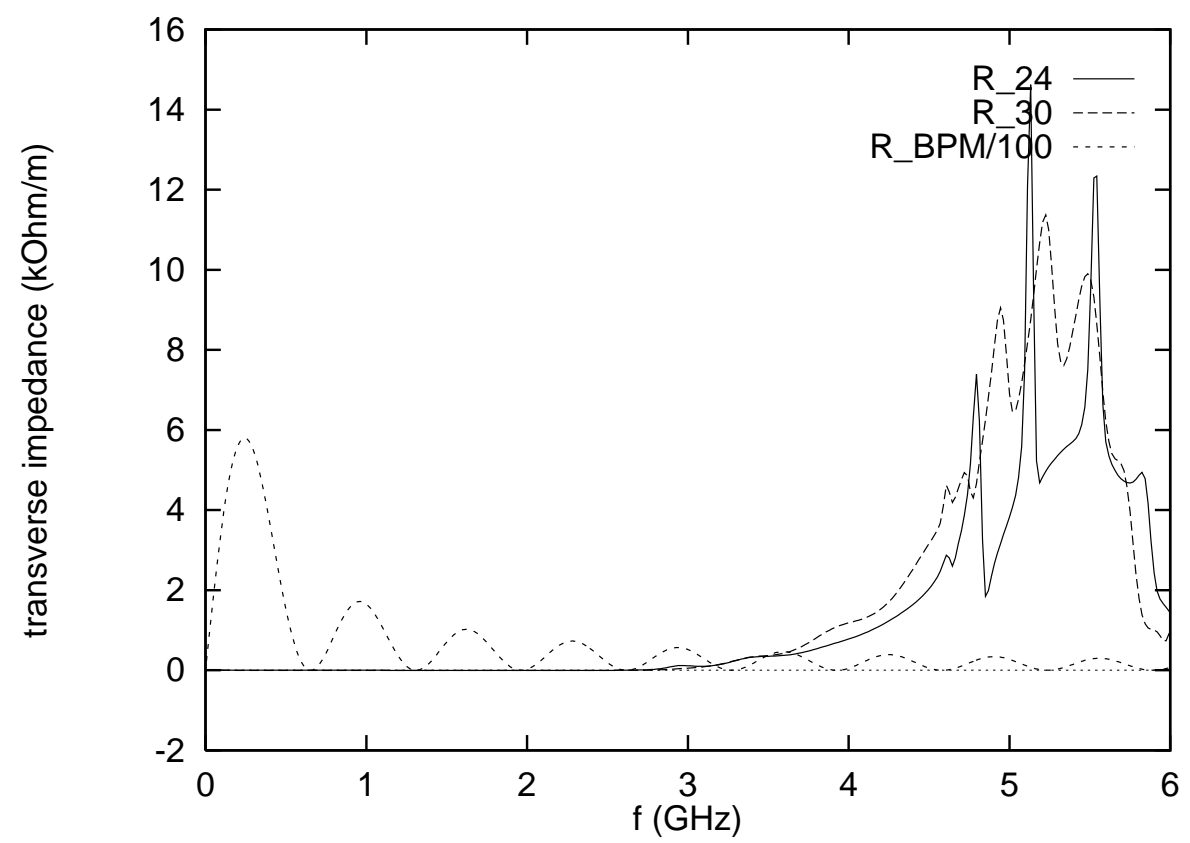

Figure 7: Transverse resistance versus frequency for the 24 and 30 convolution models. The transverse resistance of the stripline BPMs, divided by 100, is shown for comparison.

loss due to the broad band resistance. This contribution is given by

$$
P_{b b}=2 f_{b} Q_{b}^{2} \int_{f_{c u t}}^{\infty} d f F_{b}(f) R(f),
$$

where $f_{b}$ and $Q_{b}$ are the bunching frequency and charge per bunch, respectively. Using the larger form factor in Figure 5 yields $P_{b b}=12 \mathrm{~mW}$. The net power dissipation per bellows is then $P_{\text {tot }}=32 \mathrm{~mW}$ which, for 200 bellows in all, yields a $6 \mathrm{~W}$ load on the cryogenic system. There is a factor $\sim 100$ safety margin between this estimate and a noticeable load on the cryogenic system. It is interesting to note that this factor of 100 is just the increase in power one obtains by using an unphysical parabolic current pulse.

\section{Transverse Results}

Transverse impedance was estimated using the same input geometry as for the longitudinal impedance. The transverse resistance and reactance for the two bellows models are shown in Figures 7 and 8 respectively. For comparison purposes the transverse impedance of the stripline BPM system, normalized by the number of bellows, is also shown. 


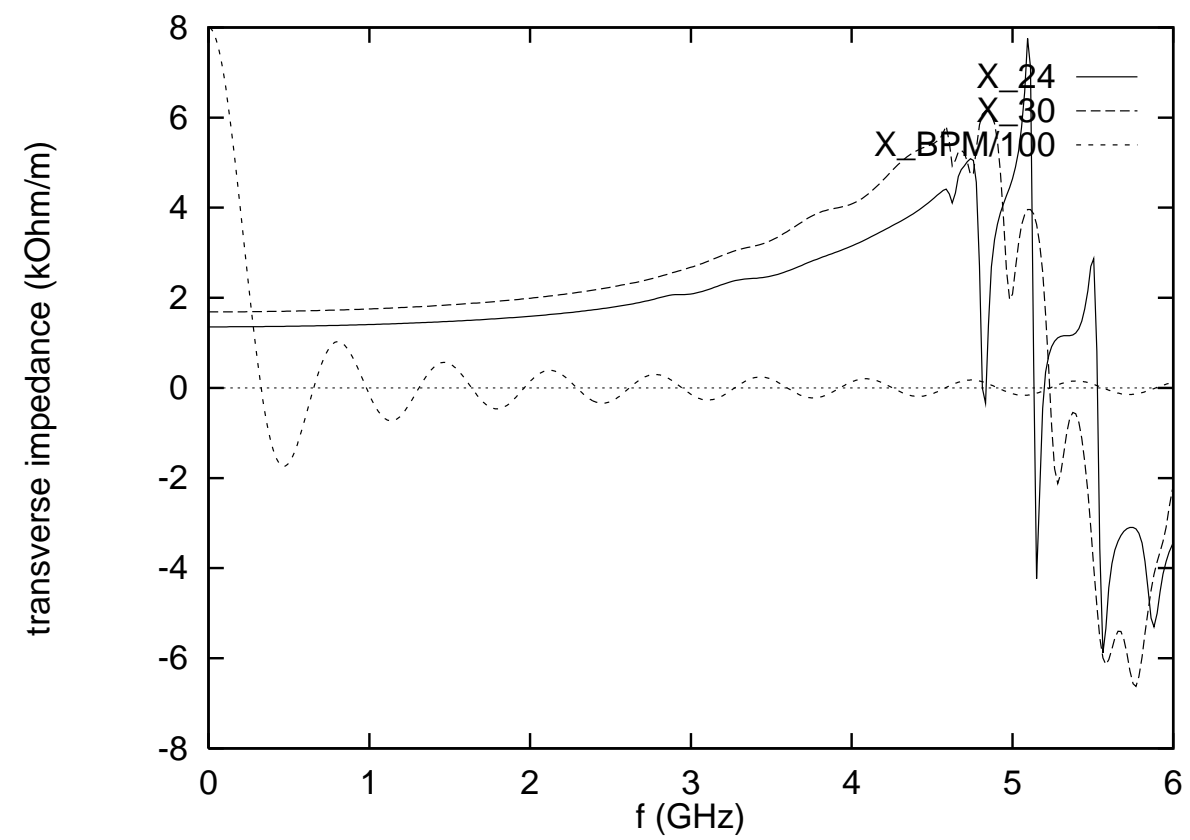

Figure 8: Transverse reactance versus frequency for the 24 and 30 convolution models. The transverse reactance of the stripline BPMs, divided by 100, is shown for comparison.

As in the longitudinal case, for dynamical purposes unshielded bellows introduce a pure reactance. For the 24 convolution case the contribution per bellows is $i 1.4 \mathrm{k} \Omega / \mathrm{m}$ which results in $i 140 \mathrm{k} \Omega / \mathrm{m}$ for 100 unshielded bellows per ring. The $\mathrm{BPM}$ contribution is 4 times larger at low frequencies. The frequency dependence of the BPM impedance is more dangerous than a pure reactance. As in the longitudinal case an exact solution exists for transverse stability with a pure reactance. The beam is always stable.

Heating due to the transverse modes will be present only if the closed orbit does not go down the center of the pipe. The heating due to the transverse mode will be comparable to the longitudinal mode heating for closed orbit distortions of order one centimeter. This is a large closed orbit distortion for high intensity operation so heating due to transverse modes will be negligible.

\section{References}

[1] Y.H. Chin, User's Guide for ABCI Version 8.7, CERN SL/94-02 (AP) (1994).

[2] Comparison of the Bellows Impedance Calculation with Measurement, V. Mane, RHIC/AP/7 (1993). 
[3] Collective Instabilities in RHIC, Editors S. Peggs and W.W. MacKay, RHIC/AP/36 (1994).

[4] D. Neuffer, Stability of Self-Consistent Longitudinal Phase-Space Distribution Under Space Charge Pertubations, Particle Accelerators 11 p23 (1980).

[5] J.D. Jackson, Classical Electrodynamics, pg 359, Wiley, (1975).

[6] G. McIntyre private communication 